\title{
APRENDIZAGEM NA INDÚSTRIA DE SOFTWARE: A INVESTIGAÇÃO DE UM HACKATHON INTERNO
}

Gisele Caroline Urbano Lourenço; Rafaela de Campos Benatti Gonçalves²; Gustavo Miguel de Oliveira ${ }^{3}$, Nelson Tenório ${ }^{1}$

${ }^{1}$ Centro Universitário Maringá - UniCesumar, Programa de Pós-Graduação Stricto Sensu em Gestão do Conhecimento nas Organizações, Maringá, PR. Bolsista CAPES. ${ }^{2}$ Universidade Estadual de Maringá - UEM, Curso de Administração, Maringá, PR. ${ }^{3}$ Centro Universitário Maringá - UniCesumar, curso de Engenharia de Software, Maringá, PR. E-mail: gisele urbano@hotmail.com.

\section{RESUMO}

Os hackathons são eventos que promovem o estímulo à criatividade e inovação. Dessa forma, muitas organizações da indústria de software têm realizados seus próprios hackathons com diferentes objetivos como, por exemplo, o de aprimorar o conhecimento individual e corporativo. Este artigo tem como objetivo investigar a aprendizagem dos indivíduos por meio de um hackathon. Para tanto, é realizada uma pesquisa de campo conduzindo questionários aos participantes de um hackathon de uma organização de desenvolvimento de software e realizou-se também uma reunião de retrospectiva com os participantes do evento para coletar as suas impressões e sugestões acerca do hackathon. Os resultados apontam que os hackathons funcionam como um espaço para criação do conhecimento dentro da organização. Além de promoverem o aprendizado organizacional, o qual impulsiona o aprendizado individual. Por fim, os hackathons criam conhecimentos que requerem os processos, ferramentas e práticas da Gestão do Conhecimento.

Palavras-chave: hackathons, aprendizagem organizacional, aprendizado individual, aprendizagem corporativa, gestão do conhecimento.

\section{LEARNING IN THE SOFTWARE INDUSTRY: INVESTIGATING AN INTERNAL HACKATHON}

\begin{abstract}
Hackathon is a sort of event which fosters the creativity and the innovation. In this way, many software industry organizations have organized their hackathons mainly addressed to improve the individual and organizational knowledge. Thus, this paper aims to investigate the individual's learning after hackathon participation. For that, we conducted a questionnaire with the participants of an internal hackathon carried out by a software development organization. Furthermore, we analyzed a retrospective meeting in which the participants gave their opinion and suggestion regarding the event. Our results point out that hackathons are considered local for knowledge creation which stimulates individual and organizational learning, driving the own learning. Finally, the hackathon creates organizational knowledge which requires Knowledge Management processes, practices, and tools to support that knowledge.
\end{abstract}

Keywords: hackathons, organizational learning, individual learning, organizational learning, knowledge, management 


\section{INTRODUÇÃO}

Uma das estratégias organizacionais para incentivar a criação de novos conhecimentos é a realização de eventos do tipo hackathons. Esses eventos incentivam o aprendizado individual e organizacional promovendo a inovação (BRISCOE; MULLIGAN, 2014). Tanto o aprendizado individual quanto o aprendizado organizacional são fundamentais para estimular o conhecimento nas organizações (SHRIVASTAVA, 1983). O conhecimento, então, é considerado um dos ativos organizacionais mais valiosos. Dessa forma, a organização que gerencia o seu conhecimento, se beneficia dele e tem maior possibilidade para inovar produtos e serviços, se mantendo sustentável no mercado em que atua (NONAKA; TOYAMA; KONNO, 2000). A Gestão do Conhecimento (GC) é indispensável para o estimulo da inovação nas organizações. A GC é compreendida como uma coleção de processos que governam a criação e disseminação do conhecimento para alcançar os objetivos organizacionais (DALKIR, 2005). Portanto, o objetivo desta pesquisa é investigar a aprendizagem dos indivíduos por meio de um hackathon realizado em uma organização de desenvolvimento de software. Este artigo está organizado em seis seções. Além desta introdução, a segunda seção apresenta os conceitos relacionados a hackathons, aprendizagem organizacional e GC. A terceira seção apresenta os procedimentos metodológicos empregados para esta pesquisa. A seção seguinte descreve o cenário de um hackathon. A quarta seção apresenta os resultados obtidos nesta pesquisa seguida das discussões. Por fim, a sexta seção apresenta as conclusões seguida das referências bibliográficas utilizadas.

\section{HACKATHONS}

Os hackathons são maratonas de programação com objetivo de solucionar determinado desafio que pode ir além do mundo tecnológico (VIVANCO-GALVÁN; CASTILLO-MALLA; JIMÉNEZGAONA, 2018). De acordo com Richterich (2017), os hackathons estimulam a criatividade dos participantes, que têm a oportunidade de lidar com a tecnologia. Os hackathons são realizados em um local, durante 24 horas, nas quais os participantes executam seus projetos. Nos hackathons os participantes têm a oportunidade de desenvolver um conteúdo interativo, pois, de acordo com o deafio proposto no evento, existe a comunicação com outros indivíduos, possibilitando então insights para a criação de determinado conteúdo (SERRANO-LAGUNA et al., 2015). Portanto, os hackathons se propõem a estimular a inovação uma vez que os indivíduos compartilham ideias e buscam soluções para os desafios apresentados. No âmbito das organizações de desenvolvimento de software os hackathons possibilitam que os funcionários gerem novos insights e compartilhem conhecimento, contribuindo, assim, para a aprendizagem organizacional.

\section{APRENDIZAGEM ORGANIZACIONAL}

A aprendizagem organizacional é um processo dinâmico que visa o desenvolvimento de capacidades e recursos de aprendizagem para aprimorar o desempenho da organização, uma vez que promove a criação contínua de novos conhecimentos (DE CUFFA et al., 2016; PÉREZ et al., 2005; WANG; AHMED, 2003). Entretanto, para qualificar uma organização como criadora de conhecimento é necessário que ela tenha a capacidade de adquirir, acumular e explorar esses conhecimentos (NONAKA; TOYAMA; KONNO, 2000). Dessa forma, Fleury e Fleury (1997) salientam que as organizações devem utilizar pressupostos que sustentem e impulsionem uma cultura de aprendizagem. Portanto, as organizações devem permitir-se aprender por meio de suas interações e ambientes. O processo de aprendizagem pode ser realizado em termos de aspectos, de processos e de produtos, que são agregadas à competência da organização (KENSKI, 2005). Entretanto, é importante que as organizações sejam orientadas a aprendizagem, onde são estabelecidos um conjunto de valores organizacionais que influenciam a criação e utilização do conhecimento (STEIL; BARCIA; PACHECO, 1999). Nesse sentido, existe uma relação clara entre aprendizagem organizacional, inovação e competitividade (PÉREZ LÓPEZ; MONTES PEÓN; 
VAZQUEZ ORDÁS, 2005). Para tanto, os processos da GC auxiliam no aprendizado organizacional uma vez que sistematizam o conhecimento a ser aplicado em produtos e serviços oferecidos pelas organizações.

\section{A GESTÃO DO CONHECIMENTO NAS ORGANIZAÇÕES}

O conhecimento é definido como uma informação combinada com experiência, contexto, interpretação e reflexão, sendo, portanto, criado a partir de um processo anterior (DAVENPORT; ECCLES; PRUSAK, 1998). O processo de criação do conhecimento precisa de um contexto físico, como um ambiente, para que se criem novos conhecimentos (NONAKA; TOYAMA; KONNO, 2000). Portanto, surge a GC com o objetivo de gerar riqueza a partir do conhecimento presente nas organizações (BUKOWITZ; WILLIAMS, 2002). Assim, a GC, a partir de seus modelos, ciclos, práticas e ferramentas, permite às organizações armazenar, codificar e compartilhar o conhecimento de maneira construtiva para que gerem conhecimento (DALKIR, 2005). Assim, o uso da GC nas organizações é essencial para que essas sistematizem o seu conhecimento, alcançando o aprendizado organizacional e possibilitando vantagens competitivas no mercado em que atuam.

\section{METODOLOGIA}

Esta pesquisa é de natureza aplicada, com objetivo exploratório em que o procedimento adotado é o método misto explanatório sequencial sugerido por Creswell (2017). O trabalho foi realizado em três fases sendo que, na primeira fase, foi efetuada busca por publicações científicas referente do tema proposto nas seguintes bases de dados on-line: Emerald Insight, Web of Science, Science Direct, Google Scholar e Portal de periódicos da CAPES. Para isso fez-se uso das seguintes palavras-chave em Língua Portuguesa: Gestão do Conhecimento; Aprendizagem Organizacional; Eventos Hacking; Hackathon. Igualmente, foram pesquisadas as seguintes palavras-chave em Língua Inglesa: Knowledge Management; Organizational Learning; Hacking Events; Hackathon. Na segunda fase foi em conduzido um questionário para coletar o grau de satisfação dos participantes de um hackathon realizado por uma organização da indústria de software. O questionário considerava seis temas. O Quadro 1 apresenta os temas doquestionário, as suas questões e os seus objetivos. As respostas obedeceram uma escala de Likert adaptada com cinco graus: (1) Ótimo, (2) Bom, (3) Regular, (4) Ruim e (5) Péssimo. Na terceira fase foi realizada uma reunião de retrospectiva, facilitada pelo gestor do hackathon, em que os participantes expuseram as suas opiniões e sugestões acerca do evento. Finalmente, a análise dos dados foi conduzida por meio do cálculo dos percentuais das respostas ao questionário que estavam tabulados em uma planilha. Além disso, os pesquisadores analisaram empiricamente as anotações tomadas durante a reunião de retrospectiva. 
Quadro 1. Questionário proposto

\begin{tabular}{|c|c|c|c|}
\hline № & Tema & Enunciado & Objetivo \\
\hline 1 & $\begin{array}{l}\text { Organização do } \\
\text { evento }\end{array}$ & $\begin{array}{l}\text { Como você avalia o } \\
\text { planejamento da organização } \\
\text { para o hackathon? }\end{array}$ & $\begin{array}{ll}\text { Obter a percepção } & \text { do } \\
\text { participante em relação ao } & \text { a } \\
\text { planejamento } & \text { do } \\
\text { hackathon. } & \\
\end{array}$ \\
\hline 2 & Desafio do evento & $\begin{array}{l}\text { Como você avalia a explicação } \\
\text { do desafio proposto para ser } \\
\text { solucionado durante o evento? }\end{array}$ & $\begin{array}{lc}\text { Investigar a } & \text { percepção do } \\
\text { participante } & \text { acerca do } \\
\text { desafio } & \text { (problema) } \\
\text { proposto. } & \end{array}$ \\
\hline 3 & $\begin{array}{ll}\text { Aspectos do } \\
\text { planejamento }\end{array}$ & $\begin{array}{l}\text { Qual a sua avaliação sobre o } \\
\text { planejamento da sua equipe } \\
\text { para a realização do evento? }\end{array}$ & $\begin{array}{l}\text { Obter a avaliação do } \\
\text { participante acerca do } \\
\text { planejamento da sua } \\
\text { equipe para a participação } \\
\text { do hackathon. }\end{array}$ \\
\hline 4 & Autoavaliação & $\begin{array}{l}\text { Qual a sua percepção em } \\
\text { relação a sua participação no } \\
\text { planejamento do evento? }\end{array}$ & $\begin{array}{ll}\text { Obter a autoavalição } & \text { do } \\
\text { participante em relação ao } & \text { a } \\
\text { planejamento } & \text { do } \\
\text { hackathon. } & \\
\end{array}$ \\
\hline 5 & Agenda do evento & $\begin{array}{l}\text { Como você avalia a agenda da } \\
\text { realização do hackathon? }\end{array}$ & $\begin{array}{l}\text { Investigar se, segundo os } \\
\text { participantes, o tempo } \\
\text { proposto para a realização } \\
\text { do hackathon estava } \\
\text { adequado. }\end{array}$ \\
\hline 6 & $\begin{array}{l}\text { Comprometimento } \\
\text { do participante }\end{array}$ & $\begin{array}{l}\text { Qual o seu grau de } \\
\text { comprometimento } \\
\text { participação do hackathon? }\end{array}$ & $\begin{array}{ll}\text { Determinar o grau } & \text { de } \\
\text { comprometimento } & \text { do } \\
\text { participante em relação ao } \\
\text { hackathon realizado. }\end{array}$ \\
\hline
\end{tabular}

Fonte: elaborado pelos autores (2018)

\section{DESCRIÇÃO DO CENÁRIO.}

Em 02 de fevereiro de 2018, a organização Accellog, especializada em desenvolvimento de softwares para a logística de transportes terrestres e situada no norte do Paraná, mais precisamente na cidade Maringá, resolveu organizar uma hackathon interno com os objetivos de capacitação e integração da equipe, além da valorização da cultura organizacional. Para tanto, o desafio proposto pelo hackathon estava relacionado ao atendimento interno da organização, pois, havia uma grande demanda de suporte de nível básico, com relação a dúvidas simples acerca das funcionalidades de um dos sistemas comercializados pela organização. Esses chamados tomavam muito tempo dos desenvolvedores em atendimento aos clientes, fato esse que diminuía a produtividade da organização. Nesse contexto, em equipes, os participantes deveriam criar soluções que buscassem diminuir o número de chamados de nível básico. Solicitou-se então, um software que automatizasse esses atendimentos de forma que o cliente pudesse interagir naturalmente e fosse redirecionado para um atendente somente em casos não-previstos anteriormente, otimizando o processo operacional do atendimento. Embora o hackathon tenha sido aberto para a participação de todos os funcionários da organização, o enfoque do evento foi o de aprimorar o conhecimento dos seus desenvolvedores e apenas eles tiveram interesse em participar. Considerando o tempo de experiência dos desenvolvedores de software que participaram do evento, essa variou entre seis meses e dez anos, demonstrando a grande diferença técnica entre os participantes. Com o intuito de organizar e estruturar o hackathon para 
garantir a entrega da solução ao desafio proposto dentro do tempo disponível, foi organizada uma agenda do evento com atividades como: recepção dos participantes, planejamento das equipes, apresentação dos desafios do hackathon, bem como a execução e a apresentação dos projetos.

\section{RESULTADOS}

Com o intuito de identificar as percepções dos participantes em relação ao hackathon, conduziu-se um questionário a cada um dos cinco participantes. Dessa forma, considerando a organização do evento, $80 \%$ dos respondentes consideraram o evento como 'ótimo' e $20 \%$ classificaram-no como 'bom'. Quando questionados a respeito da explicação do desafio do evento, os participantes foram unânimes em suas respostas, classificando-o como 'ótimo'. Em relação aos aspectos do planejamento, i.e., como cada equipe se planejou durante o evento para resolver o desafio, $40 \%$ dos participantes classificaram como 'ótimo' e $60 \%$ como 'bom'. Acerca da sua auto avaliação, i.e., o participante avalia a si próprio em relação à sua participação e comprometimento com no planejamento da sua equipe, $20 \%$ classificaram como 'ótimo' e $80 \%$ como 'bom'. Referente à agenda do evento houve convergências nas respostas dos participantes, pois $80 \%$ classificou como 'regular' e $20 \%$ classificaram como 'ruim'. Por fim, com relação ao comprometimento do participante com o evento, $40 \%$ classificaram como 'ótimo' e $60 \%$ como 'bom'. Assim, dada a ótica dos participantes, o evento teve resultados significativos para a organização. Portanto, os participantes que responderam ao questionário classificaram o evento como adequado nos mais diversos aspectos. Entretanto, os participantes acharam pouco o tempo disponível para resolver o desafio proposto pelo evento. Desse modo, observa-se que pela ótica dos participantes o evento alcançou os objetivos propostos e que para tanto, todos estavam comprometidos para a realização do hackathon. Considerando a reunião de retrospectiva realizada no mesmo dia do evento, com duração aproximada de uma hora, os participantes apontaram quatro fatores em que um evento do tipo hackathon favorece uma organização de desenvolvimento de software, sendo eles: espaço de criação, aprendizagem organizacional, aprendizagem individual e criação de novos conhecimentos. Considerando o fator 'espaço de criação', os participantes apontaram que ele possibilita a realização do trabalho de forma descontraída e interativa, em que é possível a maior troca de ideias acerca do desafio proposto. Nesse sentido, observa-se que os hackathons favorecem o compartilhamento do conhecimento. Em relação ao fator 'aprendizagem organizacional', todos os participantes afirmaram que podem aplicar os conhecimentos adquiridos no hackathon em futuros projetos organizacionais. Assim, os participantes compreenderam que o hackathon facilita a aprendizagem organizacional. Referente ao fator 'aprendizagem individual', os participantes ressaltaram que a soma do conhecimento técnico individual foi essencial para resolver o desafio apresentado. Assim, o conhecimento individual pode ser nivelado dentro das equipes. Finalmente, considerando a 'criação de novos conhecimentos', os participantes salientaram que o evento combinou o conhecimento individual com novos aprendizados, criando assim novos conhecimentos. Portanto, observa-se que a organização e seus funcionários beneficiam-se do aprendizado proporcionado pelo hackaton.

\section{DISCUSSÃO}

É importante ressaltar que os resultados desta pesquisa apontam quatro fatores importantes que se apresentam por meio da realização dos hackathons dentro das organizações. O primeiro deles a existência de um 'espaço de criação' que de acordo com Nonaka, Toyama e Konno (2000), mesmo que temporário, favorece a criação do conhecimento. Os autores ainda destacam que tal espaço de criação, é possível quando a organização fornece um contexto apropriado para facilitar as atividades em grupos, a fim de criar e acumular o conhecimento em nível individual. Nesse sentido, os hackathons se destacam por possibilitarem que os participantes se reúnam com um interesse em comum para a criação de um projeto que solucione um problema 
real (RICHTERICH, 2017). Observa-se que os hackathons são uma nova forma de trabalho na qual os funcionários têm a possibilidade de desenvolver soluções de modo descontraído e interativo possibilitando insights (SERRANO-LAGUNA et al., 2015). Portanto, hackathons devem ser utilizados como um espaço de criação, compartilhamento e inovação para o surgimento ou refinamento de ideias nas organizações. Com relação a aprendizagem organizacional oferecida pelos hackathons faz-se necessário compreender um processo dinâmico de transformação do conhecimento (STEIL; BARCIA; PACHECO, 1999). Dessa forma, os hackathons tornam-se um apoio para as organizações, pois, auxiliam na disseminação de novas técnicas ou práticas. Considerando a aprendizagem individual gerada por meio dos hackathons, nota-se que o nivelamento técnico dos funcionários da organização, que contribui para o aprendizado individual dos funcionários. Dessa forma, a aprendizagem individual é essencial para as organizações. Isto porque o processo de aprendizagem individual tem um impacto significativo no conceito e nas práticas de aprendizagem organizacional, pois, a aprendizagem começa por cada funcionário (WANG; AHMED, 2003). Contudo, as aprendizagens individuais e organizacionais devem ser alinhadas para criação e refinamento de conhecimentos (DE CUFFA et al., 2016). Por fim, a criação de novos conhecimentos possibilita inovar, produtos, processos e serviços. Entretanto, faz-se necessário a estruturação do conhecimento por meio dos modelos, ciclos, práticas e ferramentas da GC (DALKIR, 2005; NONAKA; TOYAMA; KONNO, 2000). Portanto, mesmo os hackathons possibilitando a aprendizagem por meio de seus desafios inserido em um espaço de criação faz-se necessária a GC.

\section{CONCLUSÃO}

Esta pesquisa teve como objetivo investigar a aprendizagem individual de participantes de um hackathon. Para isso, realizou-se uma pesquisa bibliográfica em bases de dados, a condução de um questionário para os participantes do evento e a análise das notas tomadas em uma reunião de retrospectiva que seguiu um protocolo de entrevistas. A partir da análise das respostas do questionário e das entrevistas, nota-se que os participantes consideram o hackathon um espaço de criação do conhecimento que contribui tanto para o aprendizado individual quanto para o aprendizado organizacional. Entretanto, faz-se necessária a GC para estruturar o conhecimento criado por meio de hackathons. Como trabalhos futuros, pretende-se aprofundar a investigação acerca da relação entre os hackathons e os processos, práticas e ferramentas da GC.

\section{REFERÊNCIAS}

BRISCOE, G.; MULLIGAN, C. Digital Innovation: The Hackathon Phenomenon. Creativeworks London, n. 6, p. 1-13, 2014.

BUKOWITZ, W. R.; WILLIAMS, R. L. Manual de gestão do conhecimento: ferramentas e técnicas que criam valor para a empresa. Porto Alegre: Bookman, 2002.

CRESWELL, J. W. Research design: qualitative, quantitative, and mixed methods approaches. Thousand Oaks: Sage publications, 2017.

DALKIR, K. Knowledge Management in Theory and Practice. Oxford: Routledge, 2005.

DAVENPORT, T. H.; ECCLES, R. G.; PRUSAK, L. Information Politics. In: The Strategic Management of Intellectual Capital. Wobourn: Butterworth-Heinemann, 1998. p. 101-120. https://doi.org/10.1016/B978-0-7506-9850-4.50010-5

DE CUFFA, D.; ZAGO, C.; ZARELLI, P. R.; RIBEIRO DE MELLO, G. O Impacto da Governança 
Corporativa Eletrônica na Gestão do Conhecimento. Navus, v. 6, n. 4, p. 98-107, 2016. https://doi.org/10.22279/navus.2016.v6n4.p98-107.421

FLEURY, A. C. C.; FLEURY, M. T. L. Aprendizagem e inovação organizacional: as experiências de Japão, Coréia e Brasil. 2. ed. São Paulo: Atlas, 1997.

KENSKI, V. W. Inovação, organização e aprendizagem organizacional. Revista científica hermes, v. 5, p. 23-37, 2005. https://doi.org/10.22279/navus.2018.v8n1.p07-19.569

NONAKA, I.; TOYAMA, R.; KONNO, N. SECl, Ba and Leadership: a unified model of dynamic knowledge creation. Long Range Planning, n. 33, p. 5-34, 2000. https://doi.org/10.1016/S0024$\underline{6301(99) 00115-6}$

PÉREZ LÓPEZ, S.; MONTES PEÓN, J. M.; VAZQUEZ ORDÁS, C. J. Organizational learning as a determining factor in business performance. Learning Organization, v. 12, n. 3, p. 227-245, 2005. https://doi.org/10.1108/09696470510592494

RICHTERICH, A. Hacking events. Convergence: The International Journal of Research into New Media Technologies, p. 1-27, 2017. DOI: 10.1177/1354856517709405

SERRANO-LAGUNA, Á.; ROTARU, D.C.; CALVO-MORATA, A.; TORRENTE, J.; FERNÁNDEZ-MANJÓN, B. Creating Interactive Content in Android Devices: The Mokap Hackaton. In: INTERNATIONAL SYMPOSIUM ON END USER DEVELOPMENT, 2015, Cham. Anais... Cham, Baviera: Springer, Cham, 2015. p. 287-290.

SHRIVASTAVA, P. A typology of organizational learning systems. Journal of Management Study, v. 20, n. 1, p. 7-28, 1983. https://doi.org/10.1111/j.1467-6486.1983.tb00195.x

STEIL, A. V; BARCIA, R. M.; PACHECO, R. C. S. An Approach to Learning in Virtual Organizations. Electronic Journal of Organizational Virtualness, v. 1, n. 1, p. 69-88, 1999.

VIVANCO-GALVÁN, O. A.; CASTILLO-MALLA, D.; JIMÉNEZ-GAONA, Y. HACKATHON Multidisciplinario: Fortalecimiento del Aprendizaje Basado en Proyectos. Revista Electrónica calidad en la educacion superior, v. 9, n. 1, p. 119-135, 2018. https://doi.org/10.22458/caes.v9i1.1893

WANG, C. L.; AHMED, P. K. Organisational learning: a critical review. The Learning Organization, v. 10, n. 1, p. 8-17, fev. 2003. https://doi.org/10.1108/09696470310457469 